\title{
TEXTURE SEGMENTATION BY THE 64X64 CNN CHIP
}

\author{
TAMÁS SZIRÁNYI \\ Analogical Comp. Lab., Comp. \& Autom. Inst., Hungarian Academy of Sci., \\ H-1111Budapest, Kende u.13-17, Hungary, sziranyi@sztaki.hu
}

\begin{abstract}
CNN's fast image processing technology helps us to run high-speed filtering tasks for image enhancement, recognition or segmentation. Texture analysis is a specific task, since the whole image is processed massively parallel while we have a limited number of texture-specific filtering and evaluation steps. Former results of simulations and recognition results of simple CNN chips show that the $\mathrm{CNN}$ is an appropriate tool for this image-processing task. Now we see what the gray-scale image processor CNN chip at its limited memory capability and data-handling/-processing accuracy can complete for multi-texture images. We demonstrate and compare some of our earlier CNNrelated texture analysis methods. Some methods to improve CNN configuration are proposed.
\end{abstract}

\section{Introduction: $\mathrm{CNN}$ for texture recognition}

Texture recognition and segmentation ask for a massively parallel computation, based on a series of simple-like (mostly early-vision effects) image processing steps. These steps are usually robust filtering and statistical evaluation effects. It is just a good role for the CNN applications. In [7,12] we see how the kernel matrices of a feedback \& feed-forward convolution system can be derived from the state equations of the network for convolution/deconvolution and nonlinear effects. This complex behavior of a single $\mathrm{CNN}$ template, combining

- convolution,

- deconvolution,

- half-toning [2],

- $\quad$ pattern-shifting [1],

- correlation between shifted state and input images,

is the crucial feature of our CNN texture analysis system. However, the parameters in the template are difficult to design since heuristics are difficult to apply. Furthermore, the processing of the combined functions is not easy to handle by analytical methods. It is important to note that the nonlinear behavior of the $\mathrm{CNN}$ can result in interesting effects that are difficult to foresee.

In [12] we presented a new single-chip texture classifier based on the Cellular Neural Network (CNN) architecture. Exploiting the dynamics of the locally interconnected 2D cell-array of a $\mathrm{CNN}$, we have developed a theoretically new method for texture classification and segmentation. This technique differs from other convolutionbased feature extraction methods since we utilize feedback convolution, and we use a genetic learning algorithm to determine the optimal kernel matrices of the network. The CNN operators we have found for texture recognition may combine different early vision effects.

The whole process included histogram equalization of the textured images, filtering with the trained kernel matrices, and decision-making based on average gray-scale or texture energy of the filtered images. We presented experimental results using digital CNN simulation with sensitivity analysis for noise, rotation and scale.

Sziranyi Texture segmentation by the 64x64 CNN chip.doc submitted to World

Scientific : 2002. 05. 03. : 15:07 
In [11] we reported a tested application performed on a programmable $22 * 20 \mathrm{CNN}$ chip with optical inputs and an execution time of a few microseconds. We have found that this CNN chip with a simple $3 * 3$ CNN kernel can reliably classify 5 textures. In [14] we found that statistical evaluation of random scanning of textures by using a filtering CNN chip can result in a high-precision recognition of up to 15 very different textures.

Texture segmentation methods can be grouped into four main classes [8]:

- Statistical methods

- Model-based methods

- Structural methods

- Methods using spatial-frequency information.

We have shown in [12] that the above methods can be partially built into a CNN filter process, which likes simple while really it is of a complex behavior. The main problem is to find the optimal parameters (templates) for optimal performance.

Since in general CNN parameters cannot be searched by linear methods (think of nonlinearity and feedback), we should be better to follow stochastic approaches, like genetic algorithm [7]. Results of [6] initiated the use of Genetic Algorithms (GA) for CNN optimization, and we show here how GAs perform on this task.

For our purposes there are three main reasons for using GAs:

- In previous applications [5], the GA performed well if there were relatively few free parameters (here, template element values) and a large amount of dependent variables (here, the output pixel values).

- There are no methods, which allow for an analytical description of textures nor how their recognition can be incorporated into the CNN structure.

- The complexity of the transient trajectories of a CNN makes it hard to trace how a slight change in the template values will influence the output. Thus, we cannot say explicitly how to modify template elements according to the difference of the desired and the computed output. However, the probabilistic operators used in GAs can overcome this difficulty.

Each template element is coded using a simple $4-8$ bit binary code in the $[-3 ; 3]$ range that corresponds to the expected VLSI technological limits. Template design methods usually consider some specific template structure [e.g. 1,2,7].

Here we make no restrictions on templates generated during optimization. Stability conditions are stated for some classes of the CNN templates [1], but using them here would unreasonably restrict the parameter space. In our case absolute stability (stable convergence) is not necessary. We need only a well-defined time-interval during which the output does not change, or changes very slightly, so that the output can be read after a pre-defined transient time. However, we have found in our experiments that our templates are usually stable in the long-term sense as well.

\section{Earlier results}

Now we see some examples of our earlier results in CNN-based texture analysis. The first results are simulation to prove that

- $\quad \mathrm{CNN}$ with feedback/feed-forward convolution kernel and nonlinear processes can achieve better and faster texture segmentation than the simple convolution-based methods (e.g. Laws-matrices);

- The limited accuracy (analog accuracy equivalent to cc. 4-6 bits of digital accuracy) is quite enough for high-precision texture analysis. 


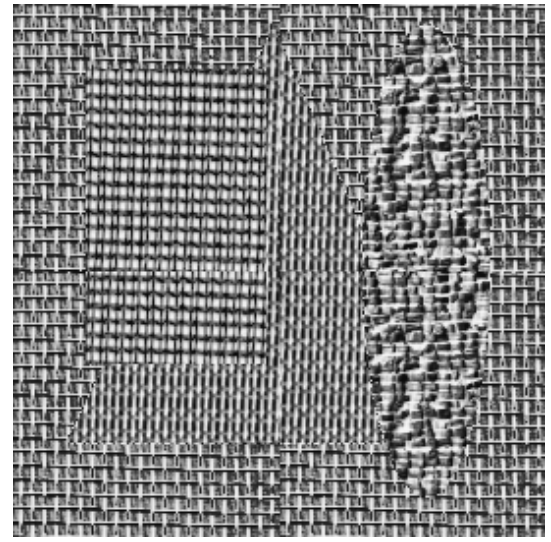

(a )

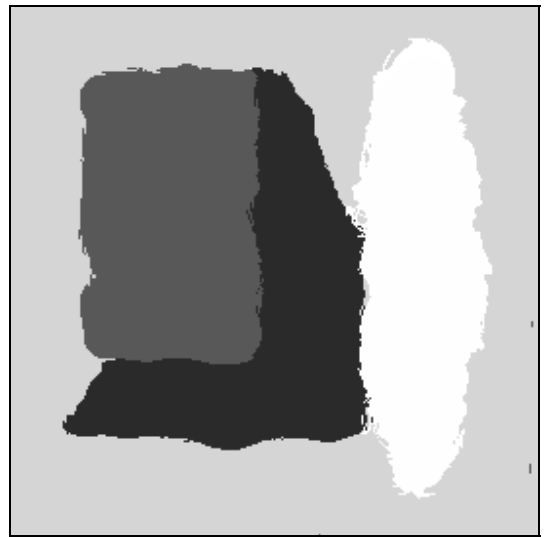

(c)

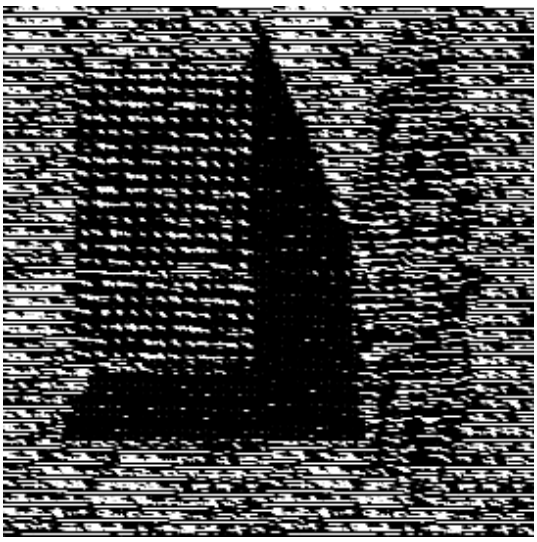

( b )

Classification error as a function of parameter accuracy

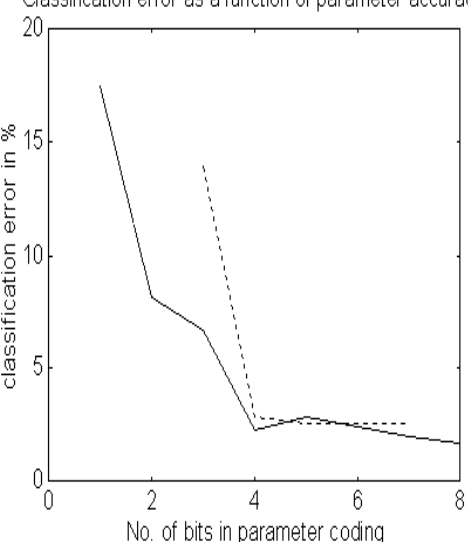

(d)

Figure 1 Simulated segmentation of four Brodatz textures using 5 different $3 * 3 \mathrm{CNN}$ templates

a) Test image $(256 \times 256)$ contains the equalized Raffia, Aluminum wire, French canvas and Straw cloth textures.

b) Halftoned-like output using a single template

c) Segmention using Bayesian-decision based on the gray-level histograms of texture outputs for each template

d) Texture misclassification error as a function of parameter accuracy. 2 different texture sets have been tested [10], using different bit-length in parameter coding. It can be seen that there is an optimal selection for bit-length assuring good convergence.

\subsection{Simulation}

The structure of the CNN-UM [9], containing template-memories and cell-memories, supports this solution. We run more templates for the same image containing multitexture patterns, and using the Bayesian-decision (or a good estimation of it) the 
classification of every pixel is done in the statistically optimum sense. The multi-template process is as follows:

- First, each detection template is run on the training image, followed by a smoothing (running the heat-diffusion process), and histograms are calculated for every texture area. In the case of 4 textures and 5 detection templates we have 20 histograms.

- In the test process, the different templates followed by the diffusion run consecutively on the test image, saving the resulting smoothed output image each time.

Finally, a Bayesian-decision is made considering the different outputs and the histograms. This process can be embedded in a hardware system containing CNN VLSI chips and some logical/arithmetic units, as for character recognition in [13]. Since simple thresholding effects can replace the histogram functions with a marginal increase in the misclassification error, the above process could be embedded in an enhanced version of a CNN-UM VLSI chip.

Fig. 1/a-c (from [12] ) show the segmentation of Raffia, Aluminum wire, French canvas and Straw cloth. The segmentation is based on the half-toned output of different $3^{*} 3$ templates. Five detection templates are used in this demonstration: 4 of these are texturespecific for one of the 4 textures and one is a 4-texture classifier. The latter yields good in other results, but this window is relatively large for segmentation. Fig. 1b shows the output of this template. Fig. 1c shows the results of segmentation. False stripes are removed and the misclassification error inside the texture areas is zero. However, the use of only 3 templates results in a moderate misclassification error.

\subsection{Robustness}

Tolerance sensitivity of template parameters is considered for VLSI implementation. Fig. $1 / \mathrm{d}$ shows the robustness-accuracy plot for 2 sets of textures when the parameters are represented for different bit-length accuracies. In other experiments it was found that the misclassification error changes only slightly in the case of image noise. These results show that the CNN process for texture detection is very robust, although the process is dynamic and the VLSI parameters may be inaccurate. This can be understood by considering the effect of the feedback: In each case of local filtering the CNN executes an error propagation process (as in half-toning [2]), which optimizes the output depending on the template characteristics and the input. This process does not accumulate the computation error, since the feedback controls the direction of the process. This means that noise and structural uncertainty do not affect the results when the CNN process itself is stable. In texture analysis, the template executes a kind of convolution / deconvolution in addition to moving effects.

\section{$2.320 \times 22 B W$ CNN chip}

We tested and early tentative-type of the CNN-UM chips. This chip [3] has a simplified structure, since its input and output are binary. It has 4 analog memories per cell. Logical functions and analog $3 * 3$ templates could be run on the chip. Template parameters are transmitted to the chip via 8 bit DA converters. The chip has optical inputs with 1 -sensor / 1-pixel scanning. This optical input array is adaptive to achieve the ratio of black pixels $(\boldsymbol{R O B})$ of around $50 \%$ in the scanned input.

In this experiment we used different Brodatz textures printed onto a transparent sheet. This transparency is mounted onto the window of the chip, and is illuminated with a 
simple table-lamp. Training and test images are real-time scanned by the on-chip sensors. The training period for 3-5 textures is only a few minutes, testing approximately 20 generations. The ROB is about $0.45-0.49$ for the input images, its small variation is independent of the texture-class. Fig. 2 shows five test images with one example of a pair of scanned-thresholded $20 * 22$ input and resulted output images for each texture. As a result, the ROB at the output is significantly different for the five texture-classes. Considering the ROB probability densities, the Bayesian-error for the recognition of the 5 textures is about $6 \%$, and it is $4-5 \%$ for 4 textures and $2-4 \%$ for 3 textures.

(a) Input textures to be scanned by the chip:
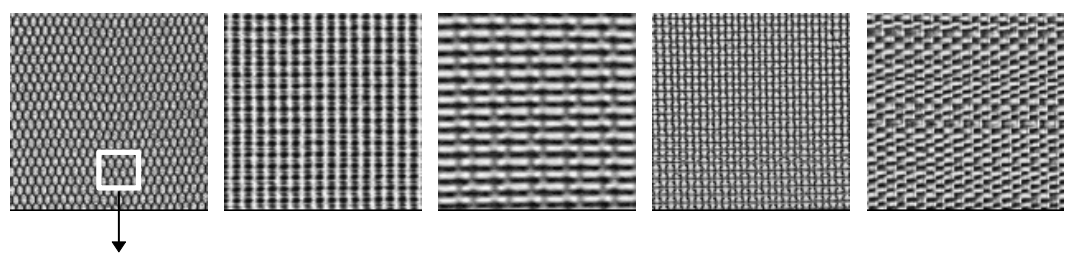

(b) Binary inputs of size $20 * 22$ in the chip-memory:
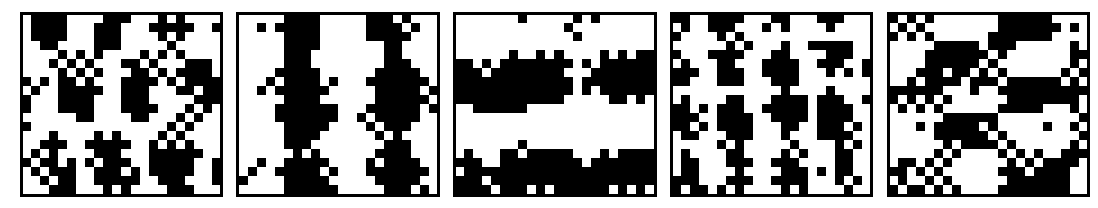

(c) Filtering results:
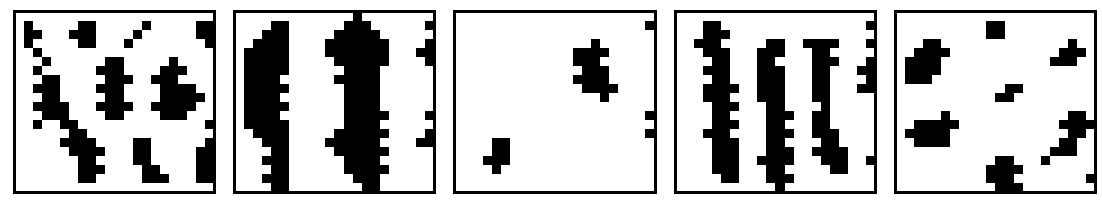

Figure 2. Classsification of 5 Brodatz textures by a programmable VLSI CNN chip of size $22 \times 20$ pixels with on-chip optical input.

a) Test images to be scanned by the on-chip photosensor-array. The texture set consists of Lizard skin, French canvas, Straw cloths and Straw matting.

b) Adaptively thresholded binary input after scanning in (with a 3.75 magnification relative to (a))

c) Binary output image after CNN transformation with different ROB for the different textures using a single $3 * 3$ template

We have also tested other texture-sets with similar results. In another experiment, we trained the chip to detect different rotated positions of textures containing nearly straight sections. The template was trained for one texture, but it can detect any other if the periods are in similar range. Applying the template and image storing capabilities of the chip, more precise recognition can be achieved in a multi-template process. Using 3 
templates to classify different sets of 4 textures (like in Fig. 2), the misclassification error is only $0.15-0.8 \%$. Rotating and tilting the image-sheet results in a slight increase of the error $(0.7-1.2 \%)$. Above we reported on the development of a single-chip texture classifier smart-sensor system, which is very fast but it has a limited window-size. We can see in [14] that this architecture can effectively recognize textures of periodicity larger than the window-size, when using statistical evaluation of the filtering output of then scanning $\mathrm{CNN}$-window. As a result, we recognized 15 Brodatz- textures by using a $20 * 22$ CNN chip with a success of $0.4 \%$ error-rate.

\section{Results on the gray-scale $64 \times 64$ chip}

The $64 x 64$ CNN chip [4] runs a gray-level analog image processing computation. This processor has much higher complexity than the previous 20x22 [3], but the higher complexity and the gray-scale data-handling may initiate unwanted effects. We should organize the main aspects of a texture segmentation task for CNN:

- Output results in half-toning effects

- To get higher contrast, more templates must be optimized together in a series

- The variance in average offset must be below $2 \%$, meaning careful biasing and transmission procedures

- The 20x22 chip had an adaptive input balancing, which is very important for stable results. Presently, it is maintained by (software) pre-equalized input images, but later adaptivity must be integrated into the hardware

- Segmentation on statistics and multi-template process is necessary for segmentation

- Histogram-based statistical decision [14] gives better results, even in unstable running conditions as well. However, the number of histogram intervals must be minimized to avoid miss-recognition coming from biasing problems.

- The template parameters are not only simple convolutions. We also incorporate the amplifier parameters of adders and multipliers. These parameters ( 2 or 3 templates together with the other chip parameters) are optimized in the genetic process together.

- Large images are processed in $64 \times 64$ blocks with 4 pixels of overlay.

In Fig. 3 we see the halftoning effect for 16 textures. First a filter template is carried out, then a symmetrical template enhance the contrast. They were optimized together. In Fig. 4 we can find a segmentation result on 4 textures with a template series of $4 \times 2$ templates trained on 16 different textures. 


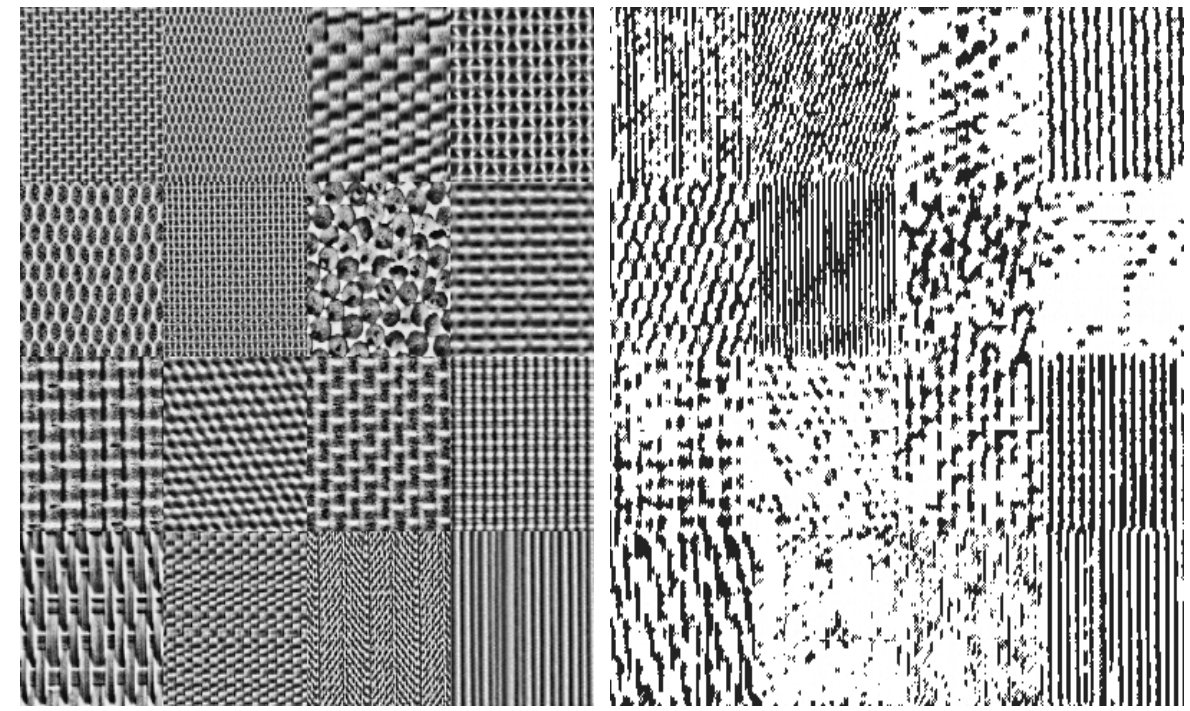

Figure 3. Original 16 textures $(400 \times 400)$ and their halftoned filtering results by the $64 \times 64$ chip, with 2 consecutive CNN templates

\section{References}

[1] L.O. Chua and T. Roska, "Stability of Class of Nonreciprocal Cellular Neural Networks", IEEE Transactions on Circuit and Systems, Vol.37, pp.1520-1527, 1990.

[2] K. Crounse, T. Roska, L.O. Chua, "Image halftoning with Cellular Neural Networks", IEEE Trans. on Circuits and Systems 40, pp.267-283, 1993.

[3] R. Domínguez-Castro, S. Espejo, A. Rodríguez-Vázquez, A. Carmona, P. Földesy, Á. Zarándy, P. Szolgay, T. Szirányi, T. Roska, “A 0.8um CMOS TwoDimensional Programmable Mixed-Signal Focal-Plane Array Processor with On-Chip Binary Imaging and Instructions Storage", IEEE J. of Solid-State Circuits, vol. 32, No. 7 pp.1013-1026, 1997.

[4] S. Espejo, R. Domínguez-Castro, G. Liñán, and Á. Rodriguez-Vázquez, “A $64 \times 64$ CNN universal chip with analog and digital I/O”, 5th Int. Conf. Electronics, Circuits and Systems (ICECS-98), Lisbon, pp. 203-206 1998.

[5] D.E. Goldberg, "Genetic Algorithms in Search, Optimization, and Machine Learning", Addison-Wesley, New York, 1993.

[6] T. Kozek, T. Roska, L.O. Chua, "Genetic algorithm for CNN Template Learning", IEEE Transactions on Circuit and Systems, Vol.40. No.6, pp.392-402, 1993.

[7] J.P. Miller, T. Roska, T. Szirányi, K. Crounse, L.O. Chua, L. Nemes, "Deblurring of Images by Cellular Neural Networks with applications to Microscopy", Proc. of 3rd IEEE Workshop on CNN and their Applications, Rome, December, pp.237242, 1994.

[8] T.R. Reed and J.M.H. Buf, "A Review of Recent Texture Segmentation and Feature Extraction Techniques", CVGIP: Image Understanding, Vol.57, pp.359-372, 1993. 
[9] T.Roska, L.O.Chua, "The CNN Universal Machine: An Analogic Array Computer," IEEE Transactions on Circuits and Systems-II, Vol. 40, March, pp. 163-173, 1993.

[10] T. Szirányi, "Robustness of Cellular Neural Networks in image deblurring and texture segmentation", Int. J. Circuit Theory and Applications, V.24, pp.381-396, May 1996.

[11] T. Szirányi, "Texture recognition using superfast Cellular Neural Network VLSI chip in real experimental environment", Pattern Recognition Letters, Vol. 18, No. 11-13, pp. 1329-1334, November 1997.

[12] T. Szirányi, M. Csapodi, "Texture Classification and Segmentation by Cellular Neural Network using Genetic Learning", Computer Vision and Image Understanding, Vol. 71, No. 3, pp. 255-270, 1998.

[13] T. Szirányi, J. Csicsvári, "High speed character recognition using a dual Cellular Neural Network architecture", IEEE Trans. Circuits and Systems, V.40, No.3(II.), pp.223231, 1993.

[14] T. Szirányi, A. Hanis, "Sub-pattern texture recognition using intelligent focal-plane imaging sensor of small window-size", Pattern Recognition Letters, Vol. 20, pp. pp.1133-1140, 1999
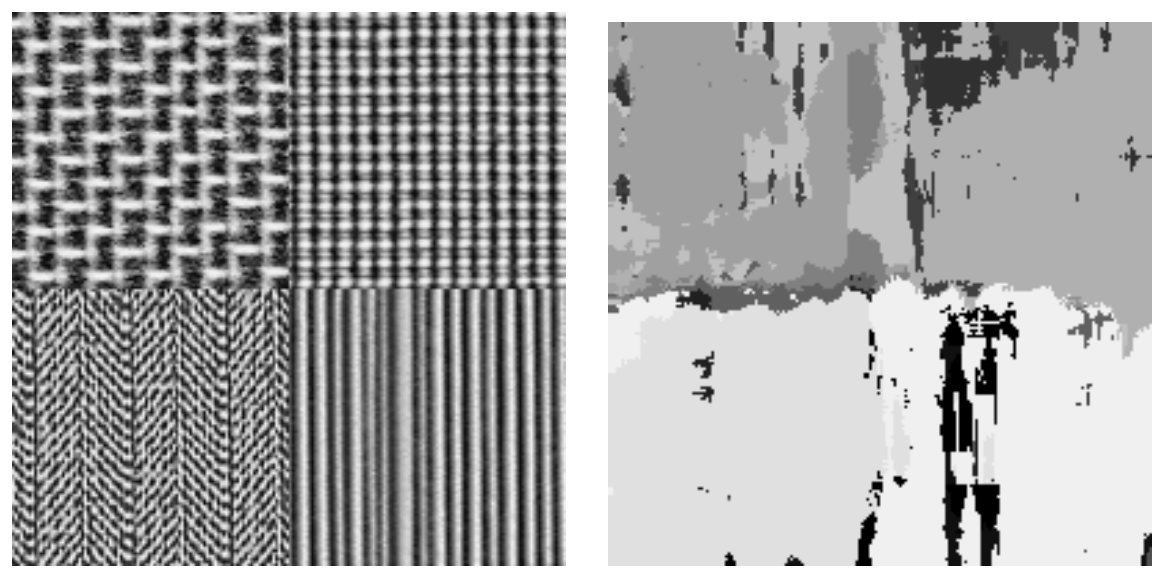

Figure 4. Multi-template segmentation results on 4 textures, trained on 16 textures 